Revue internationale P.M.E.

Économie et gestion de la petite et moyenne entreprise

\title{
Nouvelles exigences en gestion de la petite entreprise agricole : réflexions à partir du cas français
}

\section{Laurent Hemidy et Louis-Georges Soler}

Volume 5, numéro 2, 1992

URI : https://id.erudit.org/iderudit/1008140ar

DOI : https://doi.org/10.7202/1008140ar

Aller au sommaire du numéro

Éditeur(s)

Presses de l’Université du Québec

ISSN

0776-5436 (imprimé)

1918-9699 (numérique)

Découvrir la revue

Citer cet article

Hemidy, L. \& Soler, L.-G. (1992). Nouvelles exigences en gestion de la petite entreprise agricole : réflexions à partir du cas français. Revue internationale P.M.E., 5(2), 83-102. https://doi.org/10.7202/1008140ar

\section{Résumé de l'article}

Cet article propose un cadre de réflexion pour le développement de recherches dans le domaine de raide à la décision et du conseil en agriculture. Les principaux enjeux qui se présentent aujourd'hui en matière d'instruments de gestion reflètent d'une part la situation actuelle des exploitations agricoles et révolution des problèmes auxquels elles sont confrontées et d'autre part, les limites des démarches classiques de conseil auprès de ces entreprises. Compte tenu de ces enjeux, les auteurs avancent l'idée que pour aider les agriculteurs à faire face aux évolutions en cours, il faut maintenant mettre à leur disposition des outils de gestion qui tiennent compte de leurs propres logiques d'action et des contraintes qui pèsent sur leurs propres processus de décision. Suivant cette perspective, deux directions d'approfondissement peuvent structurer les contributions de la recherche : établir des modèles de fonctionnement décisionnel de l'entreprise, point de passage obligé pour la conception d'outils de pilotage appropriables par les acteurs ; réfléchir sur la place et le rôle de l'instrumentation dans la relation de conseil et contribuer à une perspective plus théorique de l'aide à la décision.
Ce document est protégé par la loi sur le droit d'auteur. L'utilisation des services d’Érudit (y compris la reproduction) est assujettie à sa politique d'utilisation que vous pouvez consulter en ligne.

https://apropos.erudit.org/fr/usagers/politique-dutilisation/ 


\title{
Nouvelles exigences en gestion de la petite entreprise agricole : réflexions à partir du cas français
}

\author{
Laurent HEMIDY \\ Louis-Georges SOLER* \\ INRA - Grignon
}

\begin{abstract}
RÉSUMÉ
Cet article propose un cadre de réflexion pour le développement de recherches dans le domaine de l'aide à la décision et du conseil en agriculture. Les principaux enjeux qui se présentent aujourd'hui en matière d'instruments de gestion reflètent d'une part la situation actuelle des exploitations agricoles et l'évolution des problèmes auxquels elles sont confrontées et d'autre part, les limites des démarches classiques de conseil auprès de ces entreprises. Compte tenu de ces enjeux, les auteurs avancent l'idée que pour aider les agriculteurs à faire face aux évolutions en cours, il faut maintenant mettre à leur disposition des outils de gestion qui tiennent compte de leurs propres logiques d'action et des contraintes qui pèsent sur leurs propres processus de décision. Suivant cette perspective, deux directions d'approfondissement peuvent structurer les contributions de la recherche : établir des modèles de fonctionnement décisionnel de l'entreprise, point de passage obligé pour la conception d'outils de pilotage appropriables par les acteurs ; réfléchir sur la place et le rôle de l'instrumentation dans la relation de conseil et contribuer à une perspective plus théorique de l'aide à la décision.
\end{abstract}

\section{ABSTRACT}

This paper gives a frame of reflection about the contribution of management research in the matter of decision support and farm advice. The principal challenge wich emerges in these days, in the matter of management tools, reflects firstly the actual situation of farms and the evolution of their problems, and

* Laurent Hémidy et Louis-Georges Soler sont chargés de recherche à l'Institut national de la recherche agronomique, à Grignon, en France. Ils poursuivent aujourd'hui leurs travaux au sein de l'unité de recherche «Instrumentation et gestion de l'entreprise ", notamment dans le domaine agricole et agro-alimentaire. Adresse : L. Hémidy - INRA ESR - 78850 Grignon, France ; L. G. Soler - INRA SAD 78850 Grignon, France. 
secondly, the limits of classical advice methods for these firms. Then the authors show that for helping farmers to face the future, we must give them management tools using the logic of action and the constraints stemming from the farmer's decision process. Following this direction, two ways could structure the future results of the research : build models of farm's decision process, important point for conception of monitoring tools usable by farmers and advisers; think and analyse the place and the role of computing tools in farm advice, and contribute to a theoretical frame of decision support.

\section{RESUMEN}

Este articulo propone un cuadro de reflexión sobre el desarrollo de investigaciones en el campo de la ayuda a la decisión y al consejo en agricultura. El principal desafio, hoy en dia, en lo que tiene que ver con los instrumentos de gestión, refleja por una parte la situación actual de las empresas agrícolas y la evolución de los problemas que tienen que enfrentar, y por otra parte los límites de las metodologias clásicas del consejo agricola. Por eso, los autores piensan que, para ayudar a los agricultores a enfrentar las evoluciones en marcha, hay que otorgarles instrumentos de gestión que toman en cuenta sus propias lógicas de acción y las coacciones influyendo sobre sus procesos de decisión. Dentro de esta perspectiva, dos direciones de analisis surgen para estructurar las investigaciones (i) establecer modelos de funcionamiento decisional de la empresa, una vía obligatoria para concebir instrumentos de pilotaje apropriables para los actores (ii) reflexionar sobre el papel de la instrumentación en la relación de consejo y contribuir a una perspectiva más teórica de la ayuda a la decisión.

\section{Introduction}

Le domaine du conseil de gestion et de l'aide aux décisions des agriculteurs connaît en France de profonds bouleversements.

Confrontées à une réduction de la population agricole et au problème du financement de leurs activités, les instances professionnelles chargées depuis plus de 20 ans de l'encadrement de l'agriculture s'interrogent sur la nature des services qu'elles doivent désormais proposer à leurs adhérents. Les centres de gestion, également confrontés au problème de leur propre développement, redéfinissent leurs stratégies en offrant de nouveaux produits : conseils de « haut niveau » auprès d'exploitations performantes, création de sociétés de service informatique, ouverture vers les petites et moyennes entreprises... Les coopératives agricoles qui ont joué un rôle important en matière d'aide à la décision dissocient, de plus en plus, vente ou achat de produits et soutien technique aux producteurs pour faire reconnaître en tant que telle cette dernière activité. Enfin, des cabinets de consultants se développent, proposant, par exemple, des « audits » d'entreprise aux exploitations de pointe. 
À ces changements importants qui vont, à terme, déboucher sur une nouvelle organisation économique du conseil en agriculture, s'ajoute la nécessité de donner un nouveau contenu aux démarches d'aide à la décision : les producteurs sont, en effet, eux aussi, confrontés à de nouvelles questions. Le développement des formules sociétaires multiplie les besoins d'aide à la décision sur les plans juridique et patrimonial. La diminution des prix des produits agricoles conduit à mettre en place une gestion beaucoup plus « fine » de l'exploitation et à rechercher un équilibre qui concilie réduction des coûts et maintien du potentiel économique de l'entreprise. Surtout, l'absence de perspectives claires en matière de prix, de débouchés commerciaux ou de nouvelles technologies oblige les agriculteurs à définir les orientations de leurs entreprises sans posséder une vision très nette des évolutions possibles de l'environnement.

Les changements que l'on commence à observer concernent donc autant les aspects institutionnels que la nature des problèmes à aborder pour aider les agriculteurs dans la conduite de leurs exploitations. Plusieurs éléments, nous y reviendrons, montrent qu'ils ne pourront pas se produire sans une réflexion nouvelle sur la pertinence des démarches et des méthodes utilisées.

Comment alors aborder aujourd'hui des travaux sur l'instrumentation de gestion, sur les outils d'aide à la décision dans ce milieu d'entreprises agricoles soumises à des transformations importantes? Telle est la question que nous voulons traiter dans cet article.

Il n'est bien sûr pas possible de répondre ici totalement à cette question ; mais nous voulons essayer de dégager quelques points de repère dans ce débat en montrant que l'évolution des problèmes de gestion des agriculteurs, d'une part, et celle des démarches de conseil d'autre part, rendent nécessaire la définition de nouvelles orientations de recherche. Ce faisant, c'est une contribution à une réflexion sur la nature des recherches en gestion dans un milieu de petites entreprises, particulièrement celui de l'exploitation agricole, que nous voulons essayer de proposer.

Dans cette perspective, nous allons dans un premier temps décrire rapidement ce milieu et ses évolutions actuelles. Sur cette base, nous discuterons des voies qui nous paraissent nécessaires de développer et nous montrerons, en particulier, l'importance d'un travail centré sur l'instrumentation de gestion d'une part, et sur les pratiques de conseil, d'autre part. Nous pourrons alors dans un dernier temps nous interroger sur l'éventuelle spécificité des recherches en gestion dans un tel secteur de petites entreprises. 


\section{La nécessité d'une nouvelle approche des problèmes de gestion}

\subsection{Une rupture radicale dans l'environnement des exploitations agricoles}

Les problèmes auxquels doit faire face le secteur agricole sont connus : crise de surproduction, concurrence accrue sur certaines productions avec d'autres pays, dont probablement à terme les pays de l'Est, contraintes de plus en plus fortes en matière de qualité des produits et de protection du milieu... On sait par exemple que les décisions concernant la nouvelle Politique agricole commune représentent d'importants changements. D'une part, le soutien du secteur agricole n'est plus assuré essentiellement par le soutien des prix mais aussi par des aides aux producteurs sous forme de compensation ou de primes. D'autre part, la Communauté européenne affiche ainsi sa volonté d'adhérer au courant de libéralisation des échanges préconisé à l'échelon international. De même, les négociations en cours à propos du GATT vont probablement induire une plus grande ouverture des marchés.

Outre une forte diminution prévisible du nombre d'exploitations, ce nouveau contexte entraîne des changements très profonds dans le fonctionnement et les stratégies des entreprises agricoles. Plusieurs points peuvent dès maintenant être soulignés :

- Pour compenser la chute des revenus, la première étape réside souvent dans la recherche d'une réduction des coûts : le suivi plus fin des processus de production de façon à diminuer les niveaux de consommations intermédiaires et l'allongement des durées d'utilisation des matériels constituent, par exemple, des voies largement explorées par les agriculteurs. Mais au-delà de ces actions, de nouvelles formes d'organisation, en particulier collectives, se mettent en place : le développement du travail en commun, l'achat d'équipements en groupes, la création de cercles d'échange de matériels et la mise en place de banques de salariés constituent autant de voies susceptibles de réduire les niveaux d'investissements et les charges de structure.

- Au-delà d'une généralisation du travail des femmes en dehors de l'exploitation, mouvement qui semble plutôt dépendant d'une évolution d'ensemble de la société, le développement de certaines formes de pluriactivité trace une autre voie envisagée par une part croissante de la population agricole. Ainsi, la mise en place d'activités non agricoles (entreprises de travaux publics et de transport, sociétés de services dans les grandes exploitations, activités liées au tourisme dans des exploi- 
tations de plus petite taille) semble parfois susceptible de contribuer à un maintien des revenus.

- Du fait d'une réorganisation profonde de diverses filières, la nature des relations entre exploitations et industries d'aval est également en train de changer. Des exigences plus fortes quant aux caractéristiques des produits agricoles renforcent la dépendance vis-à-vis de ces firmes. En même temps, certains agriculteurs tentent de se réapproprier une partie de la fonction commerciale par le développement de solutions parfois collectives (centrales d'achat, groupements de producteurs...).

De telles évolutions se traduisent par des questions nouvelles au niveau de la gestion des exploitations :

- De nouveaux modes de conduite des productions permettant une meilleure maîtrise de certains postes de charges sont à définir ; mais les références pour y parvenir ne sont pas toujours disponibles.

- Des modes de fonctionnement plus collectifs commencent à se mettre en place ; mais au-delà des problèmes juridiques posés par la création de sociétés, se pose la question des modalités de gestion en groupe de processus de production fortement soumis à des aléas.

- Des décisions engageant l'avenir sont à prendre malgré de fortes incertitudes sur les contraintes futures de marché et l'évolution des réglementations ; d'où la nécessaire prise en compte de la dimension stratégique, sans que les modalités possibles de telles réflexions stratégiques soient toujours bien maîtrisées par les agriculteurs.

- La mise en place de modes de fonctionnement plus souples, plus flexibles, permettant des adaptations rapides aux changements de l'environnement est désormais recherchée ; mais la réflexion sur les systèmes d'information, sur les outils de pilotage et de contrôle nécessaires pour y parvenir ne fait que commencer.

Voilà autant d'éléments qui illustrent le "virage " actuel que tente d'effectuer le secteur et qui expliquent la nécessité d'un renouvellement des façons d'appréhender les problèmes de gestion d'entreprise.

\subsection{Des outlls de gestion constrults sans référence aux contraintes de l'action}

Depuis bientôt quarante ans, différentes méthodes ou outils de calcul ont été proposés aux agriculteurs pour les assister dans leurs processus de prise de décision. 
Dans un premier temps, des techniques d'observation et d'analyse de l'entreprise agricole ont été mises au point (Chombart et al., 1963). Mais très vite, les économistes ont cherché à bâtir des modèles de décision, et des applications de recherche opérationnelle ont été réalisées sur des problèmes agricoles (Cordonnier et al., 1977). Les résultats furent probants, surtout dans des situations où les problèmes se prêtaient bien à une telle formulation. Cependant, le souci de rendre compte précisément de situations souvent fort complexes a obligé à une sophistication des modèles. D'où un certain rejet par les gestionnaires qui éprouvaient des difficultés grandissantes à maîtriser ce type d'approche privilégiant un point de vue formalisé quelque peu partiel des problèmes qu'ils rencontraient.

Les années 70 furent alors marquées par un retour à des démarches moins sophistiquées, de type " budget » (Attonaty et al., 1972). L'idée dominante n'était plus de rechercher une solution optimale, mais plutôt d'évaluer avec précision différentes solutions qui s'offraient aux yeux du décideur ; à charge pour lui d'effectuer un choix, compte tenu de ses propres critères. De fait, ces évaluations nécessitaient de nombreux calculs et la prise en compte d'une masse d'informations reflétant la situation de l'entreprise ainsi que les conséquences techniques et économiques de telle ou telle décision d'orientation. L'informatique permit d'automatiser l'élaboration de ces budgets et plusieurs instruments virent le jour (Planfi, Explore, Sim70...). L'Administration en imposa l'usage pour des dossiers d'installation ou de réorientation : en contrepartie, l'agriculteur pouvait bénéficier de prêts bonifiés ou de primes spéciales. Si l'utilisation de ces méthodes fut renforcée, corrélativement cette dimension administrative fit diminuer l'intérêt des agriculteurs. Il faut souligner que ce type de démarche est encore à l'ordre du jour et reste largement présent en matière d'élaboration de projet.

Parallèlement à cette approche se développèrent des démarches de suivi et d'évaluation portant sur la gestion d'ateliers de production. Concentrant leur action sur l'appui technique auprès des agriculteurs, les organismes de conseil ont mis en place des chaînes de traitement de l'information permettant tout à la fois le suivi des performances au niveau de l'atelier et l'analyse technicoéconomique à l'échelle d'un groupe d'exploitations, d'une petite région ou d'un département.

L'introduction de la micro-informatique et de la télématique constitue une nouvelle donne en matière d'instruments de gestion (Nicaud, 1991 ; Öhlmér, 1991). Cette innovation tend à rapprocher l'agriculteur du monde de l'instrumentation de gestion. Il peut désormais disposer d'outils utilisables directement. En ce qui concerne la micro-informatique, le marché du logiciel professionnel agricole, marqué par une offre pléthorique, vit une croissance relativement 
soutenue, sans pour autant se situer à la hauteur des espérances de ses promoteurs. Cependant, on peut estimer que le nombre d'agriculteurs utilisant ce type d'outil est de l'ordre de quarante mille. Les formes d'accès à l'instrument sont variées et vont du libre-service en centre de gestion à l'équipement personnel et individuel à la ferme.

Parler d'outil de gestion suppose que l'on envisage les méthodes et les techniques de gestion ainsi que les dispositifs techniques qui permettent sa mise en œuvre. À cet égard, il est indéniable que l'informatique et son évolution ont manifestement participé à la transformation des modes de diffusion des méthodes de gestion et d'aide à la décision (Hémidy, 1992). Elle apparaît de plus en plus comme le vecteur privilégié des nouvelles méthodes. Mais subsistent de nombreuses carences qui gênent les adaptations que nous avons évoquées plus haut et freinent les agriculteurs souhaitant évoluer dans leurs pratiques de gestion.

- Il faut noter, dans le mouvement général d'instrumentation de la gestion de l'exploitation agricole, la prédominance d'outils d'enregistrement et de suivi de type technique ou économique. Le caractère quasi obligatoire de la comptabilité ainsi que le large effort de diffusion réalisé par les centres de gestion expliquent en grande partie la très grande prégnance de cet outil dans les pratiques des agriculteurs. De même l'action spécialisée des différents acteurs du conseil, poussés à positionner leur action les uns par rapport aux autres, a renforcé l'usage d'outils de suivi technique ou d'outils souvent liés à une seule composante, qu'elle soit technique, financière ou trésorière. De multiples outils existent donc déjà, mais ils sont partiels, fragmentaires, peu reliés entre eux. Comptabilité, suivis techniques, gestionnaires de parcelles, plans de trésorerie... fournissent des informations aux agriculteurs ; mais il s'avère difficile de raccorder ces outils entre eux, ce qui ne facilite guère un pilotage global de l'entreprise.

- Au-delà du diagnostic faisant le point sur la situation de l'entreprise, de l'évaluation des actions passées, la nécessité de jalonner l'avenir et d'envisager différentes pistes d'évolution devient de plus en plus forte. Or, les démarches de type « budget » présentent l'inconvénient de simplifier, voire d'occulter toute réflexion sur le mode de gestion et sur les processus de décision qui conditionnent l'accès à une situation future souhaitée. Ils sont de ce fait peu utilisables dans une perspective de réflexion stratégique.

- La grande diffusion de certains outils est avant tout liée à leur caractère administratif ; cela ne signifie pas pour autant qu'ils jouent un rôle important dans la prise de décision. En outre, le fait que les organismes concernés aient leurs propres contraintes de rentabilité et d'efficacité 
entraîne des délais de restitution des résultats plutôt longs, amoindrissant alors leur intérêt pour les agriculteurs, en regard de l'aide à la décision.

- La production de références économiques destinées aux agriculteurs a longtemps été envisagée dans le cadre des " analyses de groupes ». Il s'agit d'analyses comparées des résultats d'exploitations passant par la recherche de corrélations entre caractéristiques de structures et performances ; corrélations susceptibles de définir, pour une région donnée, les « bons » ratios de structure et de productivité. Le problème est que souvent les écarts liés à des différences dans les comportements de gestion sont supérieurs aux écarts liés aux caractéristiques structurelles. Et peu de démarches sont aujourd'hui mises en œuvre pour dépasser ces difficultés.

Au total, si les actions menées pour améliorer la gestion des exploitations agricoles ont eu des effets positifs (les transformations importantes du secteur au cours des trente dernières années en sont la preuve), elles sont désormais insuffisantes pour franchir une nouvelle étape. S'il fallait résumer l'enjeu, on pourrait dire que les outils de gestion destinés aux agriculteurs ont, le plus souvent, été conçus en fonction des logiques propres des organismes de conseil. Pour aider les agriculteurs à faire face aux évolutions actuelles, il faut maintenant mettre à leur disposition des outils de gestion qui tiennent compte de leurs propres logiques d'action et des contraintes qui pèsent sur leurs propres processus de décision. Autrement dit, il faut concevoir des outils de gestion pertinents du point de vue même du pilotage de l'entreprise agricole.

\subsection{Des démarches classiques de consell qui atteignent leurs limites}

Les organismes de conseil occupent une place importante dans les transformations du monde agricole. Chargés d'aider les agriculteurs dans la gestion de leurs entreprises, ils ont depuis de longues années développé des actions d'appui technique ou de méthode, de formation, d'information, ou de sensibilisation aux conditions économiques de la production. Le milieu, et c'est là une spécificité importante, est donc très organisé et très encadré.

$\mathrm{Au}$ début des années 60 , les organismes de conseil ont pris progressivement de l'importance en cherchant à favoriser le développement de la comptabilité et des techniques de gestion (calculs de marges, prix de revient...). Mais à partir du début des années 70, la " vague » comptable, puis fiscale, qui répondait à des contraintes réglementaires, a occulté progressivement les besoins d'aide à la gestion, à tel point que dans de nombreux départements, le conseil 
de gestion n'était plus qu'une simple extension de l'approche comptable; dans certains cas, ce conseil a simplement disparu. En même temps que les divers organismes impliqués dans le conseil se spécialisaient, interdisant progressivement toute approche globale du conseil, se distendaient les relations entre chercheurs et praticiens.

C'est à partir de la fin des années 70 que des chercheurs ont souligné les difficultés créées par cet éclatement des approches pour proposer des méthodes d'analyse et de diagnostic global de l'entreprise agricole. Ils soulignaient ainsi l'importance d'une prise en compte des pratiques des agriculteurs et d'une analyse de leurs projets, de leurs objectifs avant toute intervention de conseil. Le succès de ces recommandations fut assez moyen jusqu'à la fin des années 80 , où de nouveaux « profils » de conseillers, mettant en avant leur caractère généraliste, ont commencé à apparaître.

L'importance d'une prise en compte globale des problèmes de gestion de l'exploitation agricole est désormais largement reconnue. Mais pratiquement, les interventions de conseil se déroulent encore souvent selon des procédures très standardisées et très orientées par l'outil comptable.

Face à cela, les évolutions en cours conduisent à prôner l'instauration de nouvelles formes de conseil :

- non pas fondées sur la recherche de solutions et de voies de développement standard, mais prenant en compte la spécificité de chaque situation ;

- mettant en avant une compréhension des objectifs, des projets des acteurs, plutôt que les techniques de gestion et l'outil comptable ;

- privilégiant une relation de partenariat plutôt que d'assistance.

Pour de nombreux conseillers, il s'agit là d'une évolution radicale, d'un véritable changement de métier. Longtemps leur rôle a consisté en une "prise en charge » de l'agriculteur dans ses décisions, par l'apport de solutions « toutes prêtes » à des questions ponctuelles. Ces actions ont été utiles, mais l'élévation des niveaux de formation des agriculteurs, des problèmes de gestion de plus en plus complexes conduisent à envisager le conseil comme un moyen d'aider les agriculteurs à acquérir eux-mêmes une plus grande maîtrise de la gestion de leurs entreprises.

Si de nombreux partenaires se retrouvent dans une telle approche du conseil, la plupart se sentent démunis dès qu'il s'agit de la mettre en œuvre : dans quelle démarche inscrire la relation de conseil ? Quelles méthodes utiliser ? C'est aussi sur ce plan que la recherche peut apporter sa contribution. 


\section{Voies actuelles pour le renouvellement des méthodes de gestion}

Les remarques que nous venons de faire conduisent à centrer les recherches sur deux niveaux de préoccupations :

- celui, tout d'abord, de la gestion de l'entreprise agricole, en privilégiant le point de vue interne de l'entreprise et en tenant compte des contraintes « décisionnelles » qui pèsent sur l'agriculteur dès qu'il s'engage dans l'action ;

- celui des méthodes et des pratiques de conseil, parce qu'il est évident que la relation agriculteur-chercheur ne peut s'établir que dans un petit nombre de situations et que les conseillers constituent le « pivot» d'une démultiplication, d'une généralisation de nouvelles méthodes de gestion.

\subsection{Quelle "theorle " du fonctionnement de l'exploltation pour quels outils de pilotage?}

Comme le souligne C. Midler (1990), rejoignant une idée déjà évoquée par A. Hatchuel et $\mathrm{H}$. Molet (1986) à propos de l'instrumentation de l'aide à la décision d'unités de production industrielles, « ce n'est au départ ni l'information, ni la puissance des outils de modélisation qui manquent mais plutôt une capacité à sélectionner et organiser cette information selon une représentation, une "théorie de l'atelier" pertinente par rapport au problème posé ».

De la même manière, il est clair que l'évolution des pratiques de l'aide à la décision en agriculture n'est pas d'abord conditionnée par une évolution des outils, mais bien par l'établissement d'un cadre de représentation du fonctionnement décisionnel de l'entreprise agricole. À l'instar d'une théorie des organisations dans la grande entreprise, c'est bien une telle représentation qui est susceptible de servir de grille de lecture et d'interprétation des processus de gestion mis en œuvre. C'est en fonction de ce cadre de représentation que doivent être envisagés des outils de pilotage pertinents du point de vue de l'action.

Bien qu'une approche organisationnelle ne soit pas inutile lorsqu'on analyse le fonctionnement de structures collectives agricoles, ce sont, d'une façon plus générale, des processus de décision individuels qui sont à l'œuvre en matière de gestion d'exploitation. On doit donc se donner une représentation des processus de décision mobilisés par un acteur individuel, représentation qui doit répondre aux questions suivantes. 
- Comment, confronté en permanence à des décisions variées, depuis le réglage des outils jusqu'à des décisions d'orientation à long terme, l'exploitant gère-t-il la relation entre les niveaux de décision; entre les problèmes opérationnels, tactiques, stratégiques ?

- Comment met-il en relation la gestion des processus productifs et la gestion économique et financière de l'exploitation? Autrement dit, comment assure-t-il la liaison entre les raisonnements locaux et les raisonnements globaux?

- Comment résout-il les problèmes de gestion d'un système complexe confronté en de multiples points à de fortes incertitudes, portant non seulement sur l'occurrence d'événements (évolution des prix, conditions climatiques...), mais aussi sur les conséquences possibles de ces événements (effets, par exemple, des conditions climatiques sur les résultats de processus vivants jamais totalement maîtrisés) ?

- Comment, du fait de ces incertitudes, aborde-t-il les problèmes de prévision et de planification? Comment assure-t-il la cohérence dans le temps des actions qu'il réalise?

- Quelles informations, quels indicateurs utilise-t-il ? Auxquels peut-il avoir accès du fait de son engagement dans l'action?

On le voit, les problèmes à résoudre conduisent à mettre en avant la dimension cognitive des processus décisionnels (Courbon, 1983). Toutefois, on ne présentera pas ici une « théorie » du fonctionnement de l'exploitation. Mais on peut souligner quelques points qui se dégagent des recherches actuelles et qui doivent, selon nous, orienter les réflexions sur les outils de pilotage.

\subsubsection{Le problème de la conduite de processus complexes}

Les processus de production apparaissent comme des processus soumis à de fortes perturbations, dont le contrôle n'est pas toujours facile, non seulement à cause des aléas climatiques et du caractère vivant de la matière travaillée, mais aussi, parce que les indicateurs de suivi sont très contraints par l'action.

Quelques exemples. Les chercheurs ont bâti des modèles d'évolution au cours de l'année, des ressources fourragères de prairies, susceptibles de guider les actions en matière de conduite des troupeaux. Ces modèles reposent sur des variables pertinentes quand on cherche à expliquer les niveaux de production de fourrages. Mais ces variables le sont moins lorsque l'on cherche à les utiliser pour piloter ces processus. Ainsi, les agriculteurs utilisent, par exemple, des notions de nombre de jours d'avance en stocks potentiels de fourrages à partir 
d'estimations de la hauteur d'herbe sur pied, parce que ces critères sont plus simples à obtenir lorsqu'ils se trouvent engagés dans l'action (Duru et al., 1990).

De la même façon, le choix de rentrer dans une parcelle pour engager tel ou tel travail dans les champs n'est pas conditionné par l'observation des variables sur lesquelles reposent les modèles agroclimatiques, mais par l'observation d'indicateurs d'accès facile, surtout lorsqu'il faut agir vite, comme le changement de couleur du sol sous l'effet de l'assèchement de la terre (Cerf $e t$ al., 1990).

De la même façon encore, le pilotage économique de l'exploitation repose parfois plus sur une prise en compte de flux physiques ou de critères de trésorerie (niveaux mensuels des soldes bancaires, par exemple), que sur les variables prônées par les économistes de l'entreprise agricole (Chia, 1989).

Ces indicateurs utilisés par les agriculteurs sont le produit de leur formation, mais aussi de leur expérience. Ils révèlent les points autour desquels s'organisent leurs représentations des problèmes auxquels ils sont confrontés. Surtout, ces indicateurs s'inscrivent dans des modèles d'action que les agriculteurs se construisent à partir de l'expérience et qui définissent les modes de gestion qui, de leur point de vue, sont capables de leur permettre de réaliser, avec le maximum de chances, ce qu'ils cherchent à faire (Sébillote et Soler, 1990). Cependant, dans un contexte qui change vite, dans lequel les modes de gestion doivent évoluer rapidement, l'accumulation de l'expérience devient plus difficile et l'adaptation des critères de pilotage à utiliser dans l'action devient très délicate.

Un tel constat montre bien un premier enjeu des recherches : élaborer des connaissances et des modèles utiles au pilotage suppose de tenir compte de ces variables utilisées par les agriculteurs, et surtout des contraintes qui conditionnent leur choix : facilité d'accès de l'information, urgence de la décision, simplicité du calcul... Mais surtout, il faut concevoir ce travail de production de connaissances et de modèles, de telle manière qu'il puisse aider les agriculteurs dans l'adaptation permanente des indicateurs de pilotage ainsi délimités.

\subsubsection{Le problème de l'articulation local/global}

Les problèmes de coordination entre les plans local et global sont au cœur des recherches actuelles en gestion des organisations. Paradoxalement, ils devraient l'être aussi en gestion de l'entreprise agricole, même si dans de nombreux cas, l'agriculteur assure seul une partie du processus de décision.

L'agriculteur a en effet à gérer un système complexe, au sens où il intervient sur des niveaux de décision multiples, tous soumis à des " perturbations » 
de natures variées. Une des grandes difficultés auxquelles il est confronté réside donc dans le fait qu'il doit agir sur chacun de ces niveaux de façon pertinente tout en assurant une cohérence d'ensemble, alors qu'il ne peut s'interroger en permanence sur le bien-fondé des liaisons qu'il perçoit entre chacun des niveaux de décision.

Il est donc conduit à décomposer, dans le cours de l'action, la gestion de son entreprise en une série de raisonnements locaux portant sur la conduite d'ateliers de production, la gestion de trésorerie, la gestion commerciale... Ces divers raisonnements ne se trouvent reconnectés qu'à des moments particuliers de « bilan » ou lorsqu'un " signal d'alarme » se déclenche. La maîtrise de cette reconnection est loin d'être aisée et il arrive que des logiques de gestion divergentes s'installent en différents endroits de son « système », sans qu'il s'en aperçoive nécessairement.

Il apparaît alors qu'un axe important des recherches à développer porte sur la mise au point d'instruments qui pourraient aider l'agriculteur, dans le pilotage en temps réel et de façon compatible avec ses contraintes décisionnelles, à représenter et à adapter les relations qu'il établit entre ateliers de production, gestion de trésorerie, politique d'investissement...

C'est le sens de certaines réflexions actuelles sur la mise au point de systèmes d'information à destination des agriculteurs. L'objectif est de leur procurer des informations, internes ou externes, qui leur permettent d'augmenter leur vitesse de réaction, leur souplesse et leur flexibilité. La difficulté réside dans le fait que les démarches qui se dessinent risquent plus de noyer l'agriculteur sous une avalanche de données que d'enrichir véritablement ses réflexions. Encore une fois, le problème posé est bien celui du sens et de la place de ces instruments dans le processus décisionnel de l'agriculteur (Nitsch, 1991 ; Hémidy, 1992). Compte tenu des contraintes liées à l'action, comment concevoir des systèmes d'information utiles et opératoires ? En particulier, sur quelles bases faut-il envisager leur structuration dans le temps pour permettre à l'agriculteur de maîtriser l'articulation entre raisonnements locaux et raisonnements globaux sans être conduit à s'interroger sur tout en permanence ?

\subsubsection{Le problème de l'articulation entre niveaux tactique et stratégique}

L'approche classique de la gestion de l'entreprise agricole résolvait de façon assez simple la question du pilotage stratégique de l'exploitation. L'agriculteur devait se donner un objectif et une trajectoire à suivre, et contrôler ensuite le déroulement de la trajectoire par une mesure périodique de l'écart entre le prévu et le réalisé. 
Quand on analyse les processus de décision des agriculteurs, on constate que ce mode de fonctionnement est rarement mis en œuvre. Le futur n'apparaît pas à l'agriculteur sous la forme d'une trajectoire et le pilotage s'opère moins par des contrôles d'écarts ex post, que par l'estimation ex ante des chances de maintenir une situation favorable et une capacité d'adaptation. En fait, les comportements s'apparentent à ceux d'autres types de petites entreprises, dans lesquelles on souligne parfois le caractère "incrémental » de la gestion stratégique. En effet, une fois engagée dans une voie de développement, les changements radicaux d'orientation stratégique sont rares. Par contre, devient centrale la question des inflexions et des ajustements qui peuvent s'avérer nécessaires dans le cadre de cette voie de développement.

Le problème alors posé devient celui du maintien de la convergence des ajustements successifs et de leur cohérence avec le niveau stratégique. Là encore réside une difficulté en matière de pilotage de l'exploitation : assurer cette convergence suppose de voir clairement les liaisons entre les niveaux tactique et stratégique et de repérer correctement les indicateurs de " passage » entre l'un et l'autre de ces niveaux. Ce n'est pas toujours le cas.

\subsubsection{La question des usages des instruments}

Dans les exemples précédents, nous avons à chaque fois insisté sur la nécessaire conception d'instruments nouveaux, non pas pour multiplier les sources d'information ou sophistiquer la gestion de l'exploitation, mais pour aider à la mise en relation, à l'articulation, dans le pilotage même de l'entreprise, des divers niveaux de décision, des diverses composantes de l'entreprise...

La nécessité de ce passage par l'instrumentation doit être perçue comme un support à la généralisation d'une nouvelle façon de représenter le fonctionnement de l'entreprise et son pilotage. Nous reviendrons plus loin sur cette idée. Mais mettre l'accent sur l'instrumentation comme support à l'évolution des pratiques de gestion des agriculteurs n'aurait pas de sens si l'on ne développait pas en même temps des recherches sur les usages que les agriculteurs font de ces instruments. Comme le note P. Besson (1990), « ce qu'il importe de connaître, c'est le rapport d'usage qui se noue entre l'information de gestion et l'usager, et dont résulte un comportement de gestion ». Il y a là une exigence permanente qui doit orienter les travaux actuels et qui conditionne une véritable appropriation par les acteurs eux-mêmes des instruments proposés par chercheurs et concepteurs. 


\subsection{Quelle " théorie de l'Intervention " pour quelles méthodes de conseil ?}

Compte tenu de l'existence d'un dense réseau de conseillers qui aident aux décisions des agriculteurs, de leur place centrale dans les transformations du secteur, la réflexion sur les pratiques et méthodes du conseil semble être un point de passage obligé. En fait, viser l'enrichissement des modes d'action des agriculteurs, c'est aussi enrichir et faciliter la transformation des modes d'action des hommes de conseil.

Mais il est clair que l'action du conseiller subit de fortes contraintes (temps disponible, contraintes commerciales...). Comment alors guider le conseiller dans cette voie, comment l'aider à gagner du temps dans la mise en œuvre de démarches qui peuvent en consommer beaucoup ? Selon nous, par la conception d'instruments capables de servir de support à l'interaction conseiller/agriculteur, instruments qui vont alors jouer un rôle nouveau, à savoir, structurer la relation de conseil, en fournissant une " interface », des termes de passage entre savoirs " spécifiques » de l'agriculteur et savoirs " généraux » du conseiller.

Les caractéristiques du secteur (exploitations multiples et diffuses, fort encadrement de l'agriculture...) conduisent donc à développer des recherches sur ce que nous appellerons ici une ingénierie du conseil, soit par la conception d'instruments pour l'aide à la décision, soit par la définition des cahiers des charges et des exigences que devraient satisfaire de nouveaux outils pour le conseil.

En cherchant à prendre en compte les savoirs d'experts ou de spécialistes ${ }^{1}$, les travaux récents dans le domaine de l'intelligence artificielle appliquée à l'exploitation agricole vont dans ce sens. Ces expériences ont donné lieu à la conception de systèmes experts qui sont aujourd'hui utilisés par des agriculteurs et des conseillers de gestion ${ }^{2}$. Des recherches pourraient encore être menées dans ce secteur.

C'est aussi dans cette perspective que sont envisagés quelques travaux actuels, visant la construction de modèles de simulation « à base de connaissances » sur des thèmes comme l'organisation du travail, la gestion des matériels, la gestion des systèmes fourragers et la gestion stratégique. L'usage du

1. Voir par exemple les travaux de l'équipe de J.-M. Attonaty (1987) ou encore Attonaty, Levallois et Pellerin (1987).

2. Un certain nombre de systèmes experts sont aujourd'hui opérationnels et largement utilisés (diagnostic végétal, analyse financière...). Au Québec, on peut consulter les travaux de D. Pellerin (1991). 
modèle est envisagé, non seulement pour aider au choix d'une solution (« quelle décision prendre ? »), mais aussi, et surtout, pour soutenir une réflexion sur les manières de gérer, sur les indicateurs et les règles de décision de l'agriculteur (" comment prendre des décision? »). L'accent est mis avant tout sur la dimension de l'apprentissage, c'est-à-dire sur la contribution de la démarche à un travail d'explicitation, de validation ou de remise en cause par l'agriculteur, aidé de son conseiller, de ses propres règles de décision et modes de gestion (Attonaty et Soler, 1990).

On perçoit alors un autre enjeu des recherches à conduire : identifier dans la relation de dialogue qui se noue entre un conseiller et un agriculteur, quels sont les termes de passage, les « interfaces » qui, selon les thèmes abordés, peuvent structurer, d'une part, la confrontation entre les deux types de savoirs mobilisés et, d'autre part, la réflexion sur une « amélioration » des modalités de gestion de l'entreprise.

Dans notre optique, le conseiller doit trouver un « juste milieu » entre une approche « compréhensive » qui ne chercherait qu'à aider l'entreprise à mieux se comprendre et une approche normative qui mettrait en avant l'existence d'un «bon » fonctionnement sans prise en compte des caractéristiques particulières de chaque entreprise et, surtout, sans implication des acteurs eux-mêmes dans la recherche de nouveaux modes de gestion. Cela suppose de distinguer dans chaque intervention deux étapes différentes (Sébillote, 1990).

- D'une part, l'aide au diagnostic «du point de vue de l'agriculteur»: dans cette étape, l'apport du conseiller consiste surtout à aider l'acteur à « évaluer » l'écart entre ce qu'il vise et ce qu'il est en mesure de réaliser. Autrement dit, le conseiller s'inscrit dans la logique décisionnelle de l'agriculteur et, dans cette logique-là, il l'aide à relever les contradictions, les difficultés qui pèsent sur le pilotage de l'entreprise. Des modèles de simulation conçus dans cette perspective peuvent constituer des supports pour ce type de travail.

- D'autre part, l'aide au diagnostic « du point de vue de l'intervenant »: utilisant comme "leviers » les points soulevés dans le diagnostic interne, le conseiller peut alors aider l'agriculteur à s'interroger sur la pertinence, les limites de sa logique décisionnelle et de ses objectifs. C'est à cette occasion que le conseiller peut remobiliser des savoirs généraux sur l'efficacité, au regard de telle catégorie d'objectif, de tel ou tel comportement de gestion.

Derrière ce point, c'est la question du jugement des performances de l'entreprise qui se trouve posée. Il est clair que sur des questions concernant le pilotage stratégique de l'entreprise ou son mode de fonctionnement décisionnel, on ne dispose pas d'un modèle économique de référence susceptible de fonder 
une théorie normative ${ }^{3}$. Comment, malgré tout, aider l'agriculteur à prendre une distance par rapport à ses propres raisonnements de gestion? Au regard de quelle référence extérieure à sa propre logique, peut-on l'aider à transformer ses comportements de gestion et ses objectifs?

C'est là, nous semble-t-il, un point central des travaux qui devraient être développés. Deux voies sont envisageables :

- D'une part, des analyses comparatives, mais sur des bases complètement renouvelées. La construction de typologies fondées, non pas (seulement) sur des caractéristiques de structure, mais sur la caractérisation de comportements de gestion, devraient pouvoir fournir des référentiels utiles au conseil. On pourrait ainsi comparer au regard de critères de flexibilité, de vitesse de réaction... différents processus de prise de décision en matière, par exemple, de gestion des financements long terme ou d'investissement. À la différence des typologies antérieures, on mettrait donc l'accent sur un jugement économique des mécanismes " procéduraux » qui caractérisent les processus de décision des agriculteurs (nature des indicateurs utilisés, nature des anticipations, modes de planification, nature des liaisons entre raisonnements locaux...).

- D'autre part, la conception de modèles permettant de simuler des comportements de gestion-types. Des travaux dans ce sens sont en cours de réalisation dans le domaine de l'organisation du travail (Attonaty et al., 1990) et des modalités de gestion stratégique (Attonaty et Soler, 1992). Ils peuvent s'avérer utiles pour estimer les chances d'atteindre telle catégorie d'objectif (sécurité, autonomie, rapidité de réaction) en mobilisant tel comportement de gestion (défini par un ensemble de règles de décision et d'indicateurs).

\section{Conclusion}

Les axes de travail à développer concernent donc, selon nous, les points suivants :

- La construction d'une théorie du fonctionnement décisionnel de l'entreprise agricole, point de passage obligé pour la conception d'outils de pilotage appropriables par les acteurs ; un modèle dont l'objectif

3. Voir par exemple Tanguy, 1987 et sur ce même point, en matière de gestion industrielle, les travaux du groupe ECOSIP (1990). 
serait de contribuer à répondre à des questions comme celles posées par le groupe ECOSIP (1990) : " comment organiser et maîtriser une rationalité économique dans le temps concret d'un projet ? Comment relier les enjeux économiques des phases successives en l'absence de véritable prévisibilité... comment piloter en continu, comment gérer les phénomènes d'apprentissage et d'innovation? »

- La contribution à une théorie de l'aide à la décision, au sens d'une représentation 1) de la fonction de l'aide à la décision dans un processus de décision, 2) des termes de passage entre savoirs spécifiques de l'entreprise et savoirs généraux du spécialiste des problèmes de gestion; une théorie qui aurait pour but de guider et structurer les travaux en matière d'ingénierie du conseil.

- L'élaboration de modèles pour bâtir des référentiels économiques aidant au jugement des comportements de gestion, et utiles dans les relations de conseil, non pas comme normes, mais pour aider l'agriculteur à prendre de la distance par rapport à ses propres raisonnements de gestion.

Finalement, face à l'importante diversité des PME., peut-on au regard de ces différents points affirmer une spécificité des travaux dans ce milieu de petites entreprises agricoles?

Si l'on se place au niveau de l'aide au pilotage des entreprises et de la réflexion stratégique, on peut souligner certaines convergences. Aujourd'hui, comme dans d'autres secteurs, l'agriculteur doit maîtriser de façon appropriée des processus nécessairement portcurs de risques (caractère biologique de la production, intégration de l'entreprise à l'environnement...). Et comme le souligne M. Marchesnay (1991), «plus l'entreprise est petite, plus sa compétitivité est conditionnée par l'adoption de règles de gestion spécifiques ». Ainsi, le problème posé est bien celui de la mise en place d'instruments compatibles avec les contraintes de l'action.

Par contre, l'entreprise agricole semble marquer sa spécificité au moins sur les deux aspects suivants. D'une part, elle représente généralement un collectif de travail très réduit, avec des activités souvent diversifiées, sans grande spécialisation des individus. Par conséquent, la dimension cognitive des processus de prise de décision y cst encore plus importante. D'autre part, l'encadrement de l'agriculture demeure essentiel dans la transformation du monde agricole, malgré les difficultés que ce dernier rencontre aujourd'hui. C'est pourquoi la transformation du conseil aux agriculteurs est un enjeu et explique le souci d'une démultiplication des travaux réalisés sur des terrains particuliers, ce qui confêre alors une portée plus générale aux instruments et modèles conçus à ces occasions. 
De notre point de vue, on l'a dit, la question de l'instrumentation est centrale. Si l'on suit divers auteurs dans le souci actuel de réhabilitation des instruments formalisés et des modèles en gestion d'entreprise, c'est, pour notre part, parce qu'ils représentent, à la condition d'avoir été conçus dans ce sens, des vecteurs indispensables à la démultiplication de nouvelles pratiques de gestion et de conseil.

\section{Bibliographie}

ATTONATY, J.M. et al., « Les budgets automatisés : outils nombreux pour la gestion technico-économique de l'entreprise $~$ Fourrages, $n^{\circ} 51$, p. 43-63.

AtTonaty, J.M., M.H. Chatelin, J.C. Poussin et L.G. SoleR (1990), « Un simulateur à base de connaissance pour raisonner équipement et organisation du travail en agriculture », dans Economics and Artificial Intelligence, p. 291-297.

Attonaty, J.M., M.H. Chatelin et D. SchocK (1987), « Système expert et gestion des exploitations agricoles. Une expérience menée à Grignon », BTI, $n^{\circ} 424 / 425$, p. 551-561.

Attonaty, J.M., R. Levallois et D. Pellerin (1987), « Une maquette de système expert en gestion d'entreprise agricole laitière du Québec $», B T I, n^{\circ} 424 / 425$, p. 597-609.

ATtONATY, J.M. et L.G. SoleR (1990), « Des modèles d'aide à la décision pour de nouvelles relations de conseil en agriculture », Colloque SFER, nov. 1990, p. 37-45.

ATTONATY, J.M. et L.G. SOLER (1992), « Aide à la décision et gestion stratégique : un modèle pour l'entreprise agricole ", Revue Française de Gestion, n88, p. 45-54.

BESSON, P. (1990), « Le contrôle de gestion, la valeur et le contrat, une fonction industrielle en mutation», dans "Gestion Industrielle et Mesure Économique », ECOSIP, Economica.

CERF, M., PAPY, F., AUbry, C. et J.M. MeYNARd (1990), « Théorie agronomique et aide à la décision ", dans "Modélisation systémique et système agraire », INRA.

CHIA, E. (1989), « Les pratiques de gestion des agriculteurs. La gestion en quête d'une théorie ", thèse de l'Université de Dijon.

Chombart De Lauwe, J., J. Portevin et J.C. TIRel (1963), Nouvelle gestion des exploitations agricoles, Paris, Dunod.

CORDonNiER, P., R. CARLES et P. MARSAL (1977), Économie de l'entreprise agricole, Paris, Cujas.

Courbon, J.C. (1982), « Processus de décision et aide à la décision », Économies et Sociétés, série "Sciences de gestion », $\mathrm{n}^{\circ} 3$, tome XVI. 
Duru, M., A. Gibon et P.L. Osty (1990), « De l'étude des pratiques à l'aide à la décision. L'exemple du système fourrager ", dans Modélisation systémique et système agraire, INRA.

ECOSIP (1990), Gestion Industrielle et Mesure Économique, Paris, Économica.

HATCHUEL, A. et H. MOLET (1986), « Rational modelling in understanding and aiding human decision making : about two cases studies », European Journal of Operational Research, $\mathrm{n}^{\circ} 24$.

Julien, P.A. et M. MARChenay (1987), La petite entreprise, Paris, Vuibert.

JULIEN, P.A. et M. MARCHENAY (1992), « Des procédures aux processus stratégiques dans la PME », Revue Piccola Impresa, $\mathrm{n}^{\circ} 2,1992$, p. 13-41, Turin.

MARChesnay, M. (1990), «La P.M.E. : une gestion spécifique ? , Colloque SFER, novembre 1990, p. 11-17.

HÉmidy, L. (1992), "Construction d'usages et transformation des pratiques autour d'instruments de gestion agricoles informatisés », thèse de doctorat de gestion, Paris, École polytechnique.

MIDLER, C. (1990), «Instrumentation économique et mutation industrielle : les enseignements d'une recherche chez Renault », dans «Gestion industrielle et mesure économique », Groupe ECOSIP, Paris, Économica.

NiCaUd, A. (1991), « Le changement technique et ses réseaux. Le développement de l'informatique à la ferme », thèse de doctorat de gestion, Paris, École polytechnique.

NITSCH, U. (1991), "Computers and the nature of farm management », dans The Edited Proceedings of the European Seminar on Knowledge Management and Information Technology, édité par D. Kuiper et N.G. Röling, Wageningen, The Nederlands.

OHLMER, B. (1991), « On-farm computers for farm management in Sweden : potentials and problems », Agricultural Economics, 5 : 279-286.

Pellerin, D. (1991), «Proposition d'un système expert en gestion technique des troupeaux laitiers », thèse de l'Université Laval.

SÉbillote, M. (1990), « Les processus de décision des agriculteurs. Conséquences pour les démarches d'aide à la décision », dans Modélisation systémique et système agraire, INRA.

SÉBILLOTTE, M. et L.G. SOLER (1990), « Les processus de décision des agriculteurs 》, dans Modélisation systémique et système agraire, INRA.

TANGUY, H. (1987), « L'instrumentation des choix stratégiques : pour une élaboration interactive des décisions collectives », thèse de doctorat de gestion, Paris, École polytechnique. 